\title{
Photodissociation of interstellar $\mathrm{ArH}^{+}$
}

\author{
E. Roueff ${ }^{1}$, A. B. Alekseyev ${ }^{2}$, and J. Le Bourlot ${ }^{1}$ \\ ${ }^{1}$ LERMA and UMR 8112, Observatoire de Paris, place J. Janssen, 92190 Meudon, France \\ e-mail: evelyne.roueff@obspm.fr \\ 2 Fachbereich C, Physikalische und Theoretische Chemie, Bergische Universität Wuppertal, Gaußstrasse 20, 42097 Wuppertal, \\ Germany \\ e-mail: alexeev@uni-wuppertal.de \\ 3 LERMA and UMR 8112, Observatoire de Paris, place J. Janssen, 92190 Meudon, France \\ e-mail: jacques.lebourlot@obspm.fr
}

Received 17 February 2014 / Accepted 5 April 2014

\begin{abstract}
Aims. Following the recent detection of ${ }^{36} \mathrm{ArH}^{+}$in the $\mathrm{Crab}$ nebula spectrum, we have computed the photodissociation rate of ArH ${ }^{+}$ in order to constrain the physical processes at work in this environment.

Methods. Photodissociation cross-sections of $\mathrm{ArH}^{+}$were computed in an ab initio approach that includes an explicit account of spinorbit coupling.

Results. We report the photodissociation cross-section of $\mathrm{ArH}^{+}$as a function of wavelength. Photodissociation probabilities are derived for different impinging radiation fields. The photodissociation probability for a very small unshielded cloud surrounded on all sides by the standard unshielded InterStellar Radiation Field (ISRF) model is equal to $9.9 \times 10^{-12} \mathrm{~s}^{-1}$ and $1.9 \times 10^{-9} \mathrm{~s}^{-1}$ in the Crab nebula conditions. The dependence on the visual extinction is obtained by using the Meudon photon-dominated region (PDR) code, and corresponding analytical fits are provided.

Conclusions. These data will help to produce a realistic chemical network to interpret the observations. Photodissociation of $\mathrm{ArH}^{+}$is found to be moderate, and the presence of this molecular ion mainly depends on the molecular fraction.
\end{abstract}

Key words. astrochemistry - ISM: molecules - photon-dominated region (PDR)

\section{Introduction}

While $\mathrm{ArH}^{+}$has been studied in great detail from laboratory and theoretical techniques, the detection of the ${ }^{36} \mathrm{ArH}^{+}$isotopologue in the Crab nebula (Barlow et al. 2013) offers an outstanding opportunity to exploit these studies in a previously unforeseen astrophysical context. The main motivation for this article is to investigate the response of this molecular ion to the high ambient radiation field and subsequently check the chemical processes at work in the Crab nebula environment. Section 2 describes the recent ab initio studies of $\mathrm{ArH}^{+}$. We use the available information to compute photodissociation rates resulting from different shapes of the incident radiation fields as discussed in Sect. 3. We first describe the main features of photon-dominated regions (PDR) models and introduce different radiation fields to compute the photodissociation probabilities. We summarize our conclusions in Sect. 4.

\section{Ab initio study of $\mathrm{ArH}^{+}$}

The ab initio data used in the present work to analyze the $\mathrm{ArH}^{+}$photodissociation were obtained in the detailed theoretical study (Alekseyev et al. 2007) that deals with the computation of molecular potentials and transition moments of the $\mathrm{ArH}^{+}$cation. The multireference spin-orbit configuration interaction (CI) method in its $\Lambda-S$ contracted version (LSC$\mathrm{SO}-\mathrm{CI})$ was used for this purpose. It combines a multireference single- and double-excitation CI approach (MRD-CI) with relativistic effective core potentials (RECP). The LSC-SO-CI method is described comprehensively in Alekseyev et al. (2003), while technical details of the $\mathrm{ArH}^{+}$computations are given in Alekseyev et al. (2007).

The ground $\mathrm{X}^{1} \Sigma^{+}$state of $\operatorname{ArH}^{+}$correlates to the $\operatorname{Ar}\left({ }^{1} S\right)+$ $\mathrm{H}^{+}$asymptote, whereas all low-lying excited electronic states converge to the charge-transfer $\operatorname{Ar}^{+}\left({ }^{2} P^{\mathrm{o}}\right)+\mathrm{H}\left({ }^{2} S\right)$ limit. The computed spectroscopic constants for the $X^{1} \Sigma^{+}$ground state, as well as its dipole moment, are found to be in very good agreement with experiments (Johns 1984; Šurkus 2000). While the $\mathrm{ArH}^{+}$ground state is well bound thanks to the strong argon-proton interaction, the lowest excited states, $b^{3} \Pi, B{ }^{1} \Pi$ $\left(\ldots \sigma^{2} \pi^{3} \sigma^{*}\right), a^{3} \Sigma^{+}$, and $A^{1} \Sigma^{+}\left(\ldots \sigma \pi^{4} \sigma^{*}\right)$ are all repulsive in the Franck-Condon region. They possess very shallow potential minima at large internuclear distances due to the weak attraction between the $\mathrm{Ar}^{+}$cation and hydrogen. The ab initio calculations give $230-280 \mathrm{~cm}^{-1}$ for the depths of these wells for most of the triplet states and 60-90 $\mathrm{cm}^{-1}$ in the case of $\mathrm{A}^{1} \Sigma^{+}$and $\mathrm{b}^{3} \Pi_{0}^{+}$.

Computing photodissociation of the $\mathrm{ArH}^{+}$system in realistic conditions requires taking the spin-orbit $(\mathrm{SO})$ interaction in the $\mathrm{Ar}^{+}$cation into account, in which the ${ }^{2} P_{1 / 2}-{ }^{2} P_{3 / 2}$ splitting is equal to $1431 \mathrm{~cm}^{-1}$ (Saloman 2010), corresponding to an energy of $2060 \mathrm{~K}$. Together with the various potential energy curves converging to the fine structure levels of $\mathrm{Ar}^{+}$, Alekseyev et al. (2007) have computed electric dipole moments for transitions to the states responsible for the first absorption continuum ( $A$ band) of $\mathrm{ArH}^{+}$for the first time in the approach including SO coupling, as well as the corresponding extinction coefficients $\varepsilon$ expressed in $1 \mathrm{~cm}^{-1} \mathrm{~mol}^{-1}$. It has been shown that the $\mathrm{ArH}^{+}$absorption in the $A$ band may be divided into two regions. Its high-energy part peaking at $123270 \mathrm{~cm}^{-1}$ is dominated 


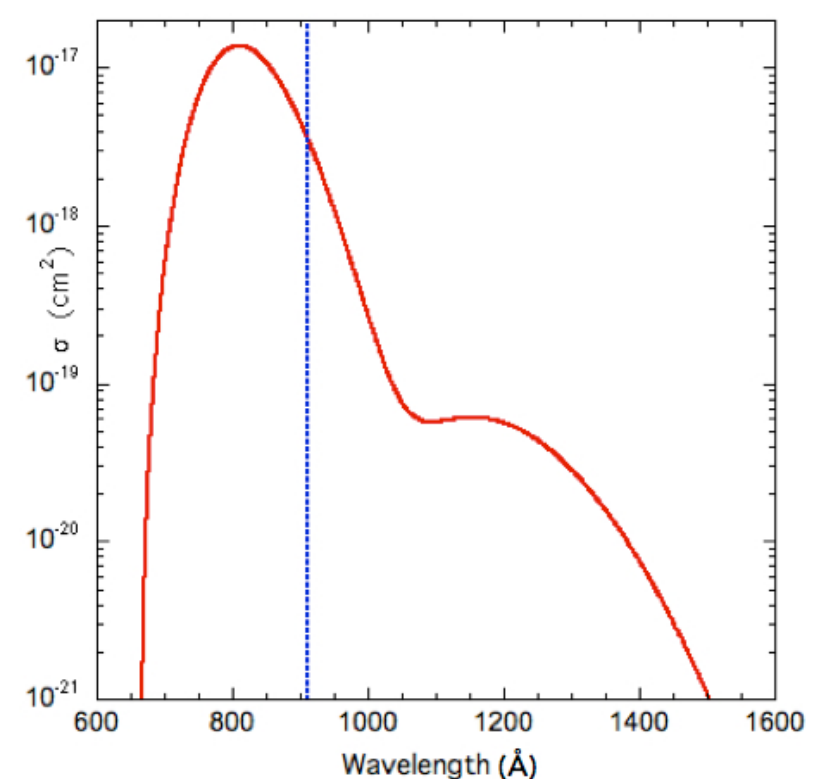

Fig. 1. Total photodissociation cross-section of $\mathrm{ArH}^{+}$as a function of incident wavelength in $\AA$. The vertical dotted blue line represents the wavelength cut-off of the interstellar radiation field

by the parallel $A^{1} \Sigma^{+} \leftarrow X^{1} \Sigma^{+}$transition, by far the strongest among transitions in this energy range. The low-energy part of the band peaking at $86560 \mathrm{~cm}^{-1}$ mainly stems from the perpendicular $B^{1} \Pi \leftarrow X^{1} \Sigma^{+}$transition, which is $\sim 240$ times weaker than $A \leftarrow X$ at its maximum. The two partial absorption spectra overlap (equal intensities at around $95 \times 10^{3} \mathrm{~cm}^{-1}$ corresponding to $1053 \AA$ ), which leads to a shoulder in the red part of the absorption curve. All other transitions in this energy region are spin-forbidden, but it can be noted that absorption into the $b^{3} \Pi_{0^{+}}$state is not negligible in the $(70-80) \times 10^{3} \mathrm{~cm}^{-1}$ range. It should be possible to detect it from the product angular distribution in the $\operatorname{Ar}^{+}\left({ }^{2} P_{3 / 2}\right)+\mathrm{H}\left({ }^{2} S\right)$ dissociation channel. The branching ratio $\Gamma$ for the final photodissociation products was also calculated and it has been shown that it smoothly increases from 0 in the red tail of the band to 1 at $E \geq 10^{5} \mathrm{~cm}^{-1}$. The latter value corresponds to the exclusive formation of the spin-excited $\operatorname{Ar}^{+}\left({ }^{2} P_{1 / 2}\right)$ ions, which can lead to the IR laser generation at the $\operatorname{Ar}^{+}\left({ }^{2} P_{1 / 2}-{ }^{2} P_{3 / 2}\right)$ transition. All spectral calculations have been carried out for ${ }^{40} \mathrm{ArH}^{+}$. It is easy to show, however, that the zeropoint-energy in the ground state of the ${ }^{36} \mathrm{ArH}^{+}$cation increases by $\sim 2 \mathrm{~cm}^{-1}$ with respect to ${ }^{40} \mathrm{ArH}^{+}$, which is of negligible importance for the present analysis.

In this study, we derive the corresponding photodissociation cross-sections from the relation $\sigma=1000 \times \log _{10}(e) \times \varepsilon / N_{\mathrm{A}}$, where $\varepsilon$ is the extinction coefficient computed in Alekseyev et al. (2007) and expressed in $1 \mathrm{~mol}^{-1} \mathrm{~cm}^{-1}$, and $N_{\mathrm{A}}$ is the Avogadro number. We thus derive $\sigma=3.82 \times 10^{-21} \times \varepsilon$, where $\sigma$ is now in $\mathrm{cm}^{2}$. Figure 1 reports the dependence of the dissociation crosssection as a function of the wavelength, expressed in $\AA$. Since all electronically excited potential curves are repulsive, the crosssection is continuous and does not display any resonance. The wavelength cut-off at $912 \AA$ takes place in astrophysical media, since all photons are absorbed if their energy is above the ionization potential of atomic hydrogen. The threshold of the total photodissociation cross-section occurs at $1500 \AA$. The values display a maximum at $800 \AA$ and become negligible below $670 \AA$.

We report the total photodissociation cross-section as a function of wavelength in the online material.
Table 1. Photodissociation cross-section of $\mathrm{ArH}^{+}$as a function of wavelength in $\AA$.

\begin{tabular}{|c|c|}
\hline $\begin{array}{c}\text { Wavelength } \\
\AA\end{array}$ & $\begin{array}{l}\text { Photodissociation cross-section } \\
\qquad \mathrm{cm}^{2}\end{array}$ \\
\hline $6.667 e+02$ & $6.880 \mathrm{e}-22$ \\
\hline $6.681 e+02$ & $1.595 \mathrm{e}-21$ \\
\hline $6.690 \mathrm{e}+02$ & $2.629 \mathrm{e}-21$ \\
\hline $6.702 e+02$ & $4.370 \mathrm{e}-21$ \\
\hline $6.711 \mathrm{e}+02$ & $6.165 \mathrm{e}-21$ \\
\hline $6.720 \mathrm{e}+02$ & $8.366 \mathrm{e}-21$ \\
\hline $6.740 \mathrm{e}+02$ & $1.499 \mathrm{e}-20$ \\
\hline $6.790 \mathrm{e}+02$ & $4.382 \mathrm{e}-20$ \\
\hline $6.802 \mathrm{e}+02$ & $5.348 \mathrm{e}-20$ \\
\hline $6.851 \mathrm{e}+02$ & $1.109 \mathrm{e}-19$ \\
\hline $6.900 \mathrm{e}+02$ & $2.033 \mathrm{e}-19$ \\
\hline $6.951 \mathrm{e}+02$ & $3.410 \mathrm{e}-19$ \\
\hline $6.970 \mathrm{e}+02$ & $4.080 \mathrm{e}-19$ \\
\hline $6.980 \mathrm{e}+02$ & $4.446 \mathrm{e}-19$ \\
\hline $7.002 \mathrm{e}+02$ & $5.363 \mathrm{e}-19$ \\
\hline $7.041 \mathrm{e}+02$ & $7.334 \mathrm{e}-19$ \\
\hline $7.081 \mathrm{e}+02$ & $9.772 \mathrm{e}-19$ \\
\hline $7.101 \mathrm{e}+02$ & $1.117 \mathrm{e}-18$ \\
\hline $7.121 \mathrm{e}+02$ & $1.271 \mathrm{e}-18$ \\
\hline $7.149 \mathrm{e}+02$ & $1.505 \mathrm{e}-18$ \\
\hline $7.170 \mathrm{e}+02$ & $1.695 \mathrm{e}-18$ \\
\hline $7.180 \mathrm{e}+02$ & $1.796 \mathrm{e}-18$ \\
\hline $7.201 \mathrm{e}+02$ & $2.009 \mathrm{e}-18$ \\
\hline $7.230 \mathrm{e}+02$ & $2.328 \mathrm{e}-18$ \\
\hline $7.240 \mathrm{e}+02$ & $2.450 \mathrm{e}-18$ \\
\hline $7.280 \mathrm{e}+02$ & $2.942 \mathrm{e}-18$ \\
\hline $7.330 \mathrm{e}+02$ & $3.645 \mathrm{e}-18$ \\
\hline $7.352 \mathrm{e}+02$ & $3.973 \mathrm{e}-18$ \\
\hline $7.390 \mathrm{e}+02$ & $4.580 \mathrm{e}-18$ \\
\hline $7.401 \mathrm{e}+02$ & $4.760 \mathrm{e}-18$ \\
\hline $7.420 \mathrm{e}+02$ & $5.081 \mathrm{e}-18$ \\
\hline $7.450 \mathrm{e}+02$ & $5.596 \mathrm{e}-18$ \\
\hline $7.470 \mathrm{e}+02$ & $5.936 \mathrm{e}-18$ \\
\hline $7.490 \mathrm{e}+02$ & $6.284 \mathrm{e}-18$ \\
\hline $7.501 \mathrm{e}+02$ & $6.486 \mathrm{e}-18$ \\
\hline $7.520 \mathrm{e}+02$ & $6.849 \mathrm{e}-18$ \\
\hline $7.555 \mathrm{e}+02$ & $7.480 \mathrm{e}-18$ \\
\hline $7.560 \mathrm{e}+02$ & $7.583 \mathrm{e}-18$ \\
\hline $7.580 \mathrm{e}+02$ & $7.949 \mathrm{e}-18$ \\
\hline $7.630 \mathrm{e}+02$ & $8.824 \mathrm{e}-18$ \\
\hline $7.700 \mathrm{e}+02$ & $1.005 \mathrm{e}-17$ \\
\hline $7.760 \mathrm{e}+02$ & $1.101 \mathrm{e}-17$ \\
\hline $7.790 \mathrm{e}+02$ & $1.144 \mathrm{e}-17$ \\
\hline $7.820 \mathrm{e}+02$ & $1.184 \mathrm{e}-17$ \\
\hline $7.870 \mathrm{e}+02$ & $1.243 \mathrm{e}-17$ \\
\hline $7.910 \mathrm{e}+02$ & $1.286 \mathrm{e}-17$ \\
\hline $7.970 \mathrm{e}+02$ & $1.332 \mathrm{e}-17$ \\
\hline $8.002 \mathrm{e}+02$ & $1.348 \mathrm{e}-17$ \\
\hline $8.102 \mathrm{e}+02$ & $1.373 \mathrm{e}-17$ \\
\hline $8.145 e+02$ & $1.370 \mathrm{e}-17$ \\
\hline $8.192 \mathrm{e}+02$ & $1.355 \mathrm{e}-17$ \\
\hline $8.260 \mathrm{e}+02$ & $1.318 \mathrm{e}-17$ \\
\hline $8.304 \mathrm{e}+02$ & $1.287 \mathrm{e}-17$ \\
\hline $8.360 \mathrm{e}+02$ & $1.238 \mathrm{e}-17$ \\
\hline $8.402 \mathrm{e}+02$ & $1.193 \mathrm{e}-17$ \\
\hline $8.452 \mathrm{e}+02$ & $1.136 \mathrm{e}-17$ \\
\hline $8.502 \mathrm{e}+02$ & $1.076 \mathrm{e}-17$ \\
\hline $8.560 \mathrm{e}+02$ & $1.002 \mathrm{e}-17$ \\
\hline $8.590 \mathrm{e}+02$ & $9.634 \mathrm{e}-18$ \\
\hline $8.702 \mathrm{e}+02$ & $8.148 \mathrm{e}-18$ \\
\hline $8.751 \mathrm{e}+02$ & $7.518 \mathrm{e}-18$ \\
\hline $8.860 \mathrm{e}+02$ & $6.154 \mathrm{e}-18$ \\
\hline $8.903 e+02$ & $5.650 \mathrm{e}-18$ \\
\hline $8.951 \mathrm{e}+02$ & $5.126 \mathrm{e}-18$ \\
\hline
\end{tabular}


Table 1. continued.

\begin{tabular}{|c|c|}
\hline $\begin{array}{l}\text { Wavelength } \\
\AA\end{array}$ & $\begin{array}{l}\text { Photodissociation cross-section } \\
\mathrm{cm}^{2}\end{array}$ \\
\hline $8.991 \mathrm{e}+02$ & $4.710 \mathrm{e}-18$ \\
\hline $9.003 e+02$ & $4.588 \mathrm{e}-18$ \\
\hline $9.052 \mathrm{e}+02$ & $4.110 \mathrm{e}-18$ \\
\hline $9.060 \mathrm{e}+02$ & $4.034 \mathrm{e}-18$ \\
\hline $9.081 \mathrm{e}+02$ & $3.843 \mathrm{e}-18$ \\
\hline $9.102 \mathrm{e}+02$ & $3.660 \mathrm{e}-18$ \\
\hline $9.106 \mathrm{e}+02$ & $3.624 \mathrm{e}-18$ \\
\hline $9.110 \mathrm{e}+02$ & $3.589 \mathrm{e}-18$ \\
\hline $9.202 \mathrm{e}+02$ & $2.871 \mathrm{e}-18$ \\
\hline $9.301 \mathrm{e}+02$ & $2.229 \mathrm{e}-18$ \\
\hline $9.401 \mathrm{e}+02$ & $1.696 \mathrm{e}-18$ \\
\hline $9.504 \mathrm{e}+02$ & $1.271 \mathrm{e}-18$ \\
\hline $9.600 \mathrm{e}+02$ & $9.565 \mathrm{e}-19$ \\
\hline $9.702 \mathrm{e}+02$ & $7.010 \mathrm{e}-19$ \\
\hline $9.802 \mathrm{e}+02$ & $5.149 \mathrm{e}-19$ \\
\hline $9.904 \mathrm{e}+02$ & $3.723 \mathrm{e}-19$ \\
\hline $9.953 \mathrm{e}+02$ & $3.187 \mathrm{e}-19$ \\
\hline $1.000 \mathrm{e}+03$ & $2.729 \mathrm{e}-19$ \\
\hline $1.005 \mathrm{e}+03$ & $2.338 \mathrm{e}-19$ \\
\hline $1.010 \mathrm{e}+03$ & $2.033 \mathrm{e}-19$ \\
\hline $1.015 \mathrm{e}+03$ & $1.743 e-19$ \\
\hline $1.020 \mathrm{e}+03$ & $1.523 e-19$ \\
\hline $1.025 \mathrm{e}+03$ & $1.319 \mathrm{e}-19$ \\
\hline $1.030 \mathrm{e}+03$ & $1.167 \mathrm{e}-19$ \\
\hline $1.035 \mathrm{e}+03$ & $1.026 \mathrm{e}-19$ \\
\hline $1.040 \mathrm{e}+03$ & $9.214 \mathrm{e}-20$ \\
\hline $1.045 \mathrm{e}+03$ & $8.351 \mathrm{e}-20$ \\
\hline $1.050 \mathrm{e}+03$ & 7.651e-20 \\
\hline $1.055 \mathrm{e}+03$ & $7.105 \mathrm{e}-20$ \\
\hline $1.060 \mathrm{e}+03$ & $6.681 \mathrm{e}-20$ \\
\hline $1.063 \mathrm{e}+03$ & $6.456 \mathrm{e}-20$ \\
\hline $1.067 \mathrm{e}+03$ & $6.238 \mathrm{e}-20$ \\
\hline $1.070 \mathrm{e}+03$ & $6.108 \mathrm{e}-20$ \\
\hline $1.073 \mathrm{e}+03$ & $6.001 \mathrm{e}-20$ \\
\hline $1.077 \mathrm{e}+03$ & $5.887 \mathrm{e}-20$ \\
\hline $1.080 \mathrm{e}+03$ & $5.826 \mathrm{e}-20$ \\
\hline $1.083 e+03$ & $5.776 e-20$ \\
\hline $1.087 \mathrm{e}+03$ & $5.738 \mathrm{e}-20$ \\
\hline $1.090 \mathrm{e}+03$ & $5.722 \mathrm{e}-20$ \\
\hline $1.093 \mathrm{e}+03$ & $5.715 e-20$ \\
\hline $1.097 \mathrm{e}+03$ & $5.719 \mathrm{e}-20$ \\
\hline $1.100 \mathrm{e}+03$ & $5.730 \mathrm{e}-20$ \\
\hline $1.110 \mathrm{e}+03$ & $5.791 \mathrm{e}-20$ \\
\hline $1.120 \mathrm{e}+03$ & $5.883 \mathrm{e}-20$ \\
\hline $1.130 \mathrm{e}+03$ & $5.974 \mathrm{e}-20$ \\
\hline $1.140 \mathrm{e}+03$ & $6.036 \mathrm{e}-20$ \\
\hline $1.150 \mathrm{e}+03$ & $6.078 \mathrm{e}-20$ \\
\hline $1.160 \mathrm{e}+03$ & $6.081 \mathrm{e}-20$ \\
\hline $1.170 \mathrm{e}+03$ & $6.032 \mathrm{e}-20$ \\
\hline $1.180 \mathrm{e}+03$ & $5.948 \mathrm{e}-20$ \\
\hline $1.190 \mathrm{e}+03$ & $5.814 \mathrm{e}-20$ \\
\hline $1.200 \mathrm{e}+03$ & $5.646 \mathrm{e}-20$ \\
\hline $1.220 \mathrm{e}+03$ & $5.210 \mathrm{e}-20$ \\
\hline $1.240 \mathrm{e}+03$ & $4.679 \mathrm{e}-20$ \\
\hline $1.260 \mathrm{e}+03$ & $4.076 \mathrm{e}-20$ \\
\hline $1.280 \mathrm{e}+03$ & $3.453 \mathrm{e}-20$ \\
\hline $1.299 \mathrm{e}+03$ & $2.880 \mathrm{e}-20$ \\
\hline $1.300 \mathrm{e}+03$ & $2.855 \mathrm{e}-20$ \\
\hline $1.320 \mathrm{e}+03$ & $2.308 \mathrm{e}-20$ \\
\hline $1.340 \mathrm{e}+03$ & $1.806 \mathrm{e}-20$ \\
\hline $1.360 \mathrm{e}+03$ & $1.384 \mathrm{e}-20$ \\
\hline $1.380 \mathrm{e}+03$ & $1.041 \mathrm{e}-20$ \\
\hline $1.400 \mathrm{e}+03$ & $7.560 \mathrm{e}-21$ \\
\hline $1.420 \mathrm{e}+03$ & $5.398 \mathrm{e}-21$ \\
\hline $1.441 \mathrm{e}+03$ & $3.721 \mathrm{e}-21$ \\
\hline
\end{tabular}

Table 1. continued.

\begin{tabular}{cc}
\hline \hline $\begin{array}{c}\text { Wavelength } \\
\AA\end{array}$ & $\begin{array}{c}\text { Photodissociation cross-section } \\
\mathrm{cm}^{2}\end{array}$ \\
\hline $1.461 \mathrm{e}+03$ & $2.519 \mathrm{e}-21$ \\
$1.490 \mathrm{e}+03$ & $1.361 \mathrm{e}-21$ \\
$1.510 \mathrm{e}+03$ & $8.606 \mathrm{e}-22$ \\
$1.521 \mathrm{e}+03$ & $6.746 \mathrm{e}-22$ \\
$1.530 \mathrm{e}+03$ & $5.386 \mathrm{e}-22$ \\
$1.550 \mathrm{e}+03$ & $3.234 \mathrm{e}-22$ \\
$1.571 \mathrm{e}+03$ & $1.862 \mathrm{e}-22$ \\
$1.591 \mathrm{e}+03$ & $1.059 \mathrm{e}-22$ \\
$1.599 \mathrm{e}+03$ & $7.594 \mathrm{e}-23$ \\
\hline
\end{tabular}

\section{Photodissociation probabilities}

We now consider $\mathrm{ArH}^{+}$photodissociation for different impinging radiation fields. As emphasized in van Dishoeck et al. (2006), photodissociation occurs in the surface layers of molecular environments exposed to UV radiation fields. We use the Meudon PDR code facility ${ }^{1}$ (Le Petit et al. 2006) to compute the photodissociation probability of $\mathrm{ArH}^{+}$for different incident radiation fields as a function of the visual magnitude.

The main feature of the Meudon PDR code is a detailed computation of the radiative transfer of the VUV radiation field in a cloud model containing both gas and dust. Photodissociation of molecular hydrogen is computed from detailed molecular properties of Lyman and Werner transitions as computed by Abgrall et al. (1993, 2000), involving a discrete absorption followed by emission toward the continuum of the $\mathrm{X}$ molecular state. Absorption by gas and dust is treated in a consistent way and we refer the reader to the reference paper describing the Meudon PDR code (Goicoechea \& Le Bourlot 2007; Le Petit et al. 2006).

\subsection{Photodissociation in an unshielded environment.}

The photodissociation (photoionization) probability of any molecular (atomic) species for a very small parcel of gas surrounded on all sides by the ISRF is expressed as

$k_{\mathrm{pd}}^{\text {cont }}=\int_{912}^{\lambda \max } \sigma(\lambda) N(\lambda) \mathrm{d} \lambda$,

where $N(\lambda)$ is the number of photons per square centimeter per second, and per $\AA$ and $\sigma(\lambda)$ the corresponding cross-section. We express this quantity as a function of the radiation energy density via

$N(\lambda)=\frac{\lambda}{4 \pi h} u(\lambda)$,

which is valid for an isotropic incident radiation field. We show in Fig. 2 the incident energy densities of three radiation fields that were used for the computations. We constrained those radiation fields to contain the same integrated incident energy density for a wavelength range between 912 and $2400 \AA$, corresponding to the Habing cut-off of the UV radiation field (Habing 1968). This choice is slightly different from the one made by van Dishoeck et al. (2006), who used a cut-off of $2050 \AA^{2}$. The model using the incident Draine radiation field is defined as the standard case.

1 Available at http://pdr.obspm.fr

2 We have verified that we obtain identical results for carbon photoionization in that case. 
Table 2. Photodissociation rates computed for an unshielded environment.

\begin{tabular}{llccc}
\hline \hline Radiation field & $\begin{array}{l}\text { Ratio of energy } \\
\text { densities }\end{array}$ & $\operatorname{Pd}\left(\mathrm{ArH}^{+}\right)\left(\mathrm{s}^{-1}\right)$ & \multicolumn{2}{c}{$\operatorname{Pi}(\mathrm{C})\left(\mathrm{s}^{-1}\right)$} \\
& 1 & $9.9 \times 10^{-12}$ & $3.1 \times 10^{-10}$ & $3.1 \times 10^{-10}$ \\
& 1 & $7.0 \times 10^{-13}$ & $1.7 \times 10^{-11}$ & $2.5 \times 10^{-11}$ \\
Draine & 1 & $3.7 \times 10^{-11}$ & $7.6 \times 10^{-10}$ & \\
Black body of 10000 K & $1.9 \times 10^{-9}$ & $4.1 \times 10^{-8}$ & \\
Black body of 37000 K & 1 & $1.9 \times 1 \times 1$ result & \\
Crab nebula & 83.9 & & &
\end{tabular}

Notes. VD06 refers to van Dishoeck et al. (2006).

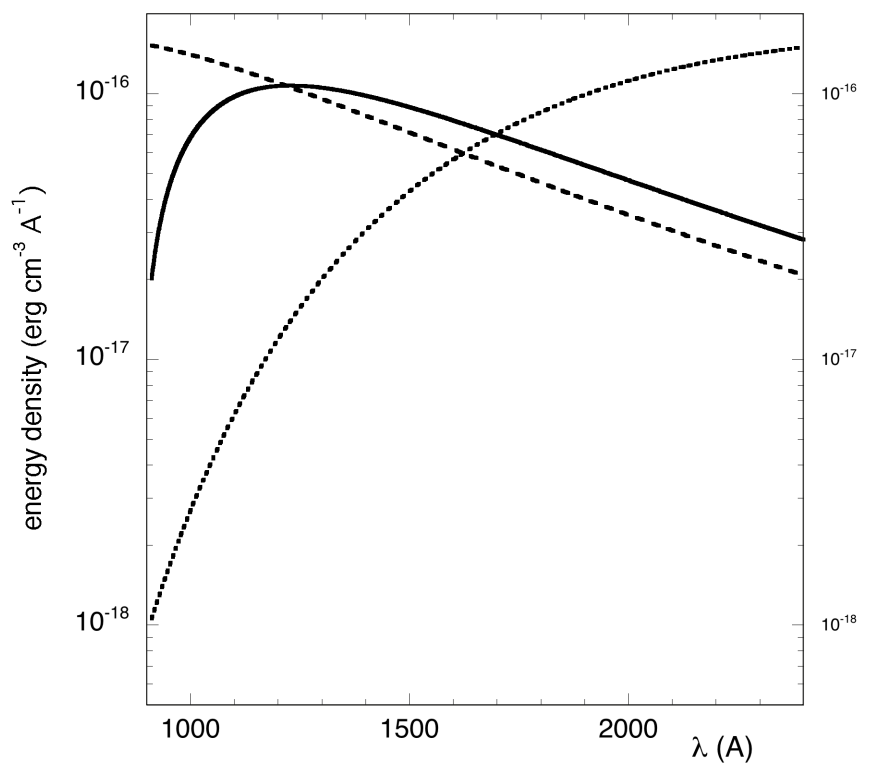

Fig. 2. Energy density of the radiation fields as a function of wavelength. Solid line: Draine, dotted line: black body at $T=10000 \mathrm{~K}$, dashed line: black body at $T=37000 \mathrm{~K}$.

The radiation field impinging the molecular knots where $\mathrm{ArH}^{+}$has been detected (Barlow et al. 2013) may be estimated as well. The pulsar wind nebula (PWN) luminosity is obtained from a dust + photoionization modeling of the Crab nebula by Owen \& Barlow (in prep.) using the spectrum displayed in Hester (2008). The integrated PWN luminosity is $1.3 \times 10^{38} \mathrm{erg} / \mathrm{s}$ and is assumed to be emitted from the central two-thirds of the ellipsoidal nebula, whose overall dimensions are adopted to be $3.8 \mathrm{pc} \times 2.9 \mathrm{pc}$. Table 2 displays the photodissociation rate $\left(P_{\mathrm{d}}\right)$ of $\mathrm{ArH}^{+}$, as well as the photoionization probability $\left(P_{\mathrm{i}}\right)$ of atomic carbon computed from the direct integration in Eq. (1) for the three chosen radiation fields and for the crab nebula environment. We also display the ratios of the radiation field energy densities to the one corresponding to the Draine radiation field in the 911-2400 $\AA$ window. The results concerning photoionization of carbon given by van Dishoeck et al. (2006) are also reported. The differences obtained reflect the different choice of normalization of the radiation fields. The value displayed for the Crab nebula is about two hundred times greater than in standard interstellar conditions, and the ratio of the corresponding energy densities is about 80 .

\subsection{Role of the dusty environment}

We consider now a cloud irradiated on one side and follow the decrease in the radiation field as it penetrates inside the molecular cloud. This reduction is a result of photon absorption both by gas and dust. Dust absorption and scattering properties are introduced using the prescription by Weingartner \& Draine (2001) for the Milky Way for spherical dust particles with radii between $0.3 \mu$ and $3 \mathrm{~nm}$ following a -3.5 power law of the dust size distribution (Mathis et al. 1977). Dust absorption is a continuum process as is gas phase absorption by $\mathrm{ArH}^{+}$, as shown in Fig. 1. We also recall that such a continuum mechanism occurs in the photoionization of carbon because the corresponding cross-section is constant from $I_{\mathrm{C}}=11.26 \mathrm{eV}$ (corresponding to $1101.5 \AA$ ), equal to $1.6 \times 10^{-17} \mathrm{~cm}^{2}$ up to $13.6 \mathrm{eV}$.

An exponential decrease is often used to represent the $A_{\mathrm{V}}$ dependence of the photoprocesses, and the corresponding parameters are found in the different kinetic databases $\left(\mathrm{KIDA}^{3}\right.$, Wakelam et al. 2012; UDFA ${ }^{4}$, McElroy et al. 2013). These expressions aim to describe the effect of dust continuum absorption on the photoionization and photodissociation probabilities. We consider a cloud model with a proton density $n_{\mathrm{H}}=10^{3} \mathrm{~cm}^{-3}$ that is submitted to a cosmic ionization rate of $5 \times 10^{-17} \mathrm{~s}^{-1}$, corresponding to standard translucent cloud conditions. The radiation, impinging isotropically on one side of a semi-infinite slab, is described by the Draine analytic ISRF model (Draine 1978). We display the computed $\mathrm{ArH}^{+}$photodissociation and carbon photoionization probabilities in Fig. 3 as a function of $A_{\mathrm{V}}$. The corresponding exponential fits are displayed in the lefthand part a of Fig. 3 for a restricted visual extinction range between 0 and 3, as recommended in van Dishoeck et al. (2006). We also display in the righthand part $b$ of Fig. 3 the corresponding fitting functions using the exponential integral $E_{2}$ function. Whereas the exponential dependence is a fair approximation of the photodissociation probabilities for the considered range of visual extinctions, the fit using the $E_{2}$ function is superior as already emphasized and used in Neufeld \& Wolfire (2009). Corresponding formulae are displayed in Table 3.

We also computed the photodissociation rate resulting from radiating stars, considered as the blackbodies already introduced in Sect. 3.1 and displayed in Fig. 2, where the radiation is considered to be beamed perpendicular to the surface of the cloud, which corresponds to regions located close to a particular star. In those cases, exponential fits are found to be adequate representations of the visual extinction $\mathrm{A}_{V}$ dependence of the photodissociation rates, as shown in Fig. 4. Finally, we also computed the photodissociation probability corresponding to the Crab nebula conditions where the radiation is isotropic. We assumed a proton density $n_{\mathrm{H}}=2 \times 10^{4} \mathrm{~cm}^{-3}$ representative of the molecular knots detected by Loh et al. (2012) in this environment. The corresponding results are displayed in Fig. 4 and fitting expressions given in Table 3 . The constant factors expressed in $\mathrm{s}^{-1}$ are about one half the value given for the unshielded case (Table 2) because the radiation is impinging on one single side of the cloud.

\footnotetext{
3 http: ://kida.obs.u-bordeaux1.fr

4 http://www .udfa.net
} 

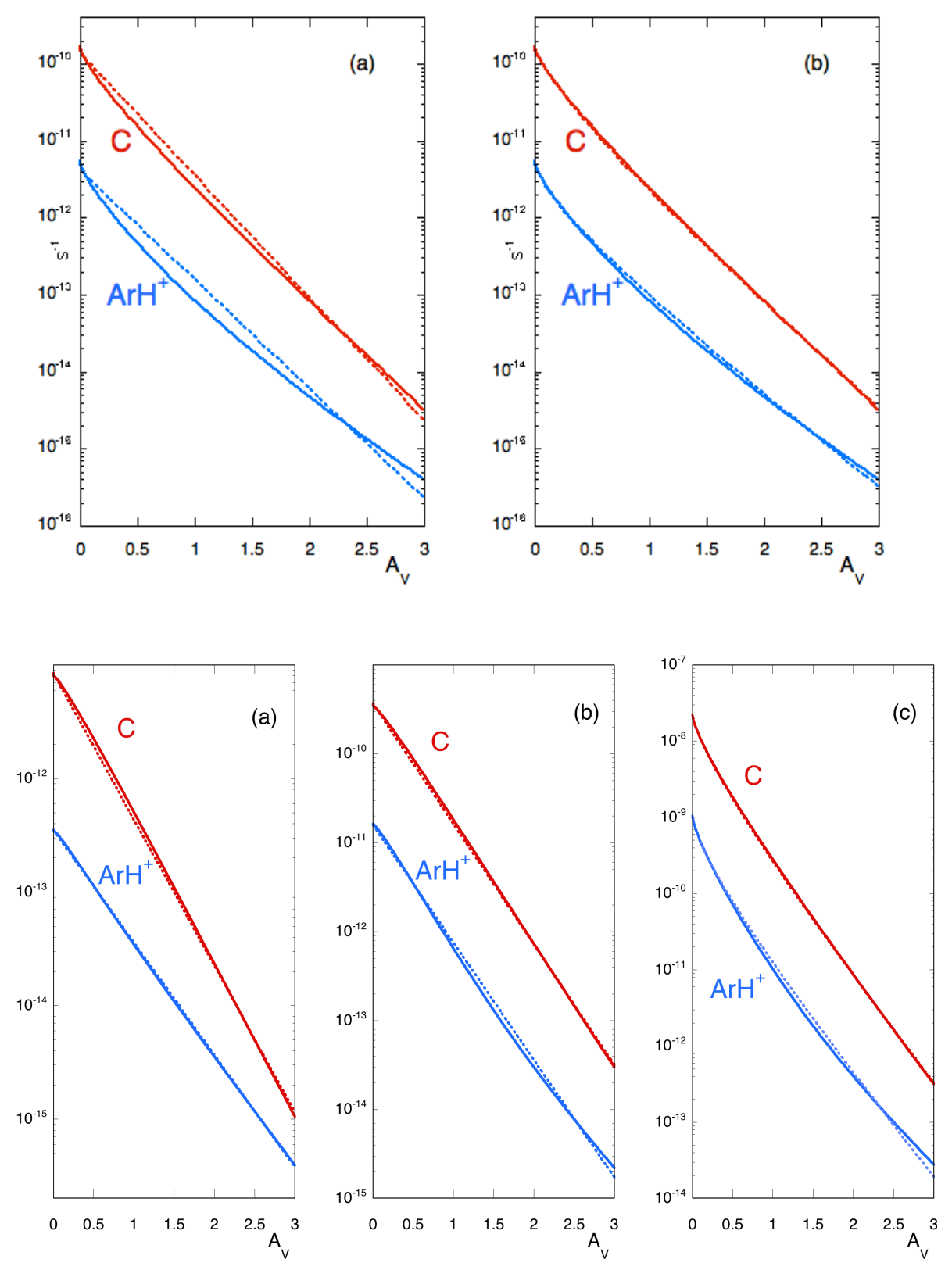

Fig. 4. Computed photodissociation and photoionization rates of $\mathrm{ArH}^{+}$(blue) and $\mathrm{C}$ (red) at the surface of a semi-infinite slab as a function of visual extinction $A_{\mathrm{V}}$. Solid lines correspond to PDR model results, and dotted lines display the fit equations results, as given in Table 3. a)-c) represent the $\mathrm{BB} 10000 \mathrm{~K}, \mathrm{BB} 37000 \mathrm{~K}$, and the Crab nebula cases.

Table 3. Fitting functions of the photodissociation rate of $\mathrm{ArH}^{+}$and photoionization rate of carbon as functions of the visual magnitude for the different radiation fields used in Table 2 .

\begin{tabular}{lll}
\hline \hline Radiation field & $\operatorname{Pd}\left(\mathrm{ArH}^{+}\right)\left(\mathrm{s}^{-1}\right)$ & $\operatorname{Pi}(\mathrm{C})\left(\mathrm{s}^{-1}\right)$ \\
\hline IS Draine & $4.2 \times 10^{-12} \times \exp \left(-3.27 A_{\mathrm{V}}\right)$ & $1.40 \times 10^{-10} \times \exp \left(-3.67 A_{\mathrm{V}}\right)$ \\
IS Draine & $4.84 \times 10^{-12} \times E_{2}\left(2.46 A_{\mathrm{V}}\right)$ & $1.78 \times 10^{-10} \times E_{2}\left(2.84 A_{\mathrm{V}}\right)$ \\
PP BB 10000 K & $3.54 \times 10^{-13} \times \exp \left(-2.28 A_{\mathrm{V}}\right)$ & $8.91 \times 10^{-12} \times \exp \left(-2.99 A_{\mathrm{V}}\right)$ \\
PP BB 37000 K & $1.56 \times 10^{-11} \times \exp \left(-3.04 A_{\mathrm{V}}\right)$ & $3.83 \times 10^{-10} \times \exp \left(-3.14 A_{\mathrm{V}}\right)$ \\
IS Crab & $9.66 \times 10^{-10} \times E_{2}\left(2.83 A_{\mathrm{V}}\right)$ & $2.29 \times 10^{-8} \times E_{2}\left(2.93 A_{\mathrm{V}}\right)$ \\
\hline
\end{tabular}

Notes. IS and PP stand for isotropic and perpendicular irradiation, respectively.

\subsection{Role of the continuous gas phase absorption}

However, these analytic representations of photodissociation/photoionization probabilities neglect the role of other gasphase continuum absorptions. Re-emphasizing early PDR models by Tielens \& Hollenbach (1985), Rollins \& Rawlings (2012) pointed out recently that neglecting mutual shielding by dust and carbon leads to overestimating the photoionization probability of carbon by a factor $\exp \left(-\sigma_{\mathrm{C}} N_{\mathrm{C}}\right)$, where $\sigma_{\mathrm{C}}$ is the photoionization cross-section of carbon and $N_{\mathrm{C}}$ stands for the column density of atomic carbon. We investigate in Fig. 5 the role of additional gas phase absorption for the standard model case, where 


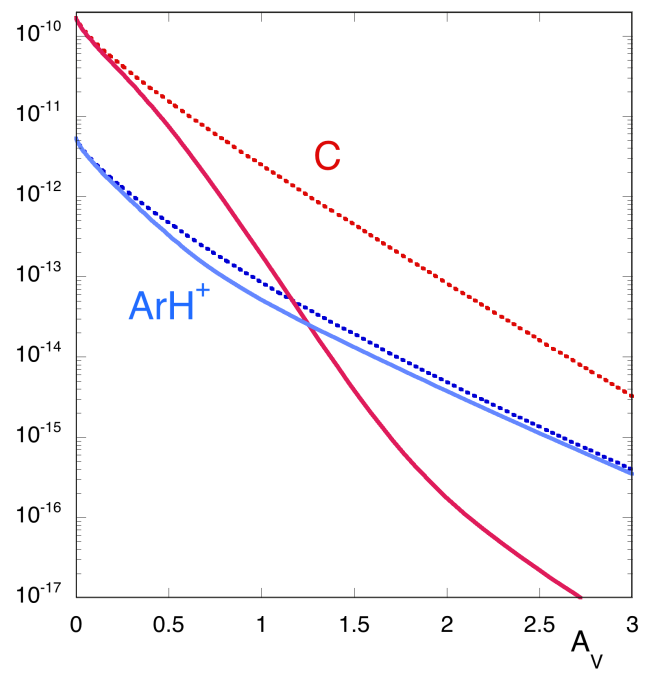

Fig. 5. Computed photodissociation and photoionization rates of $\mathrm{ArH}^{+}$ (blue curves) and C (red curves) at the surface of a semi-infinite slab for the Draine radiation field; solid line: PDR model including continuous dust + gas phase absorption; dotted line: PDR model with continuous dust absorption alone.

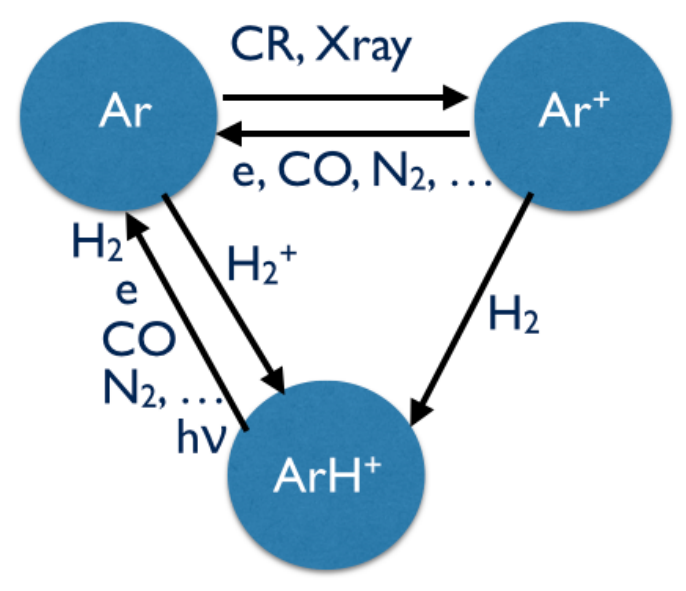

Fig. 6. Chemical network of the $\mathrm{ArH}^{+}$molecular ion.

we introduce the photodissociation and photoionization continuous cross-sections of different gas phase compounds, including Carbon. Models including continuous absorption of the radiation field by gas are compared to computations that neglect that effect. While gas phase absorption considerably modifies the photoionization probability $\mathrm{A}_{V}$ dependence for carbon, the effect on $\mathrm{ArH}^{+}$photodissociation is much less significative since the photodissociation threshold is at $1500 \AA$, at a significantly longer wavelength than for carbon photoionization (1105 $\AA$ ).

\subsection{Relevance to the Crab environment}

The main chemical processes involved in the formation and destruction of $\mathrm{ArH}^{+}$, except for photodissociation, are discussed in Barlow et al. (2013). The availability of the photodissociation cross-sections allows constraining the chemical processes at work further. The chemical network is relatively simple as displayed in Fig. 6. The initial step of the chemistry is ionization of argon through cosmic rays and X-rays followed by a reaction with molecular hydrogen. An alternative path is provided by the reaction between $\mathrm{H}_{2}^{+}$with neutral argon. The abundance of $\mathrm{ArH}^{+}$ is thus directly proportional to the total ionization rate of Ar. The ionization state of Ar is discussed by Jenkins (2013) in the context of the warm neutral medium (WNM) and non conventional sources of ionization are proposed to reconcile a $[\mathrm{ArI} / \mathrm{OI}]$ ratio that is consistent both with the observations and the generally accepted value of the density $n(\mathrm{HI})=0.5 \mathrm{~cm}^{-3}$. It is interesting to note that the molecular $\mathrm{OH}^{+}$ion, which is recognized as a test of cosmic ionization rate (Indriolo 2013), has been detected in the same environment (Barlow et al. 2013). A quantitative study is beyond the scope of the present paper, and a realistic study would require introducing the $\mathrm{X}$ ray spectrum. The photodissociation rate of $\mathrm{ArH}^{+}$at the surface of the molecular knots is computed to be about $2 \times 10^{-9} \mathrm{~s}^{-1}$, which may compete with dissociative recombination (with associated rate constant $k_{\mathrm{e}} \leq 5 \times 10^{-8} \mathrm{~cm}^{3} \mathrm{~s}^{-1}$, Mitchell et al. 2005). However, reactions with molecular hydrogen (with a rate of $\sim 2 \times 10^{-9} \mathrm{~cm}^{3} \mathrm{~s}^{-1}$, Anicich 2003) are presumably the main destruction channel.

\section{Summary}

We have reported photodissociation cross-sections of $\mathrm{ArH}^{+}$as a function of wavelength, and they can be used to compute the response of that molecular ion to ultraviolet interstellar radiation fields. The photodissociation rate of $\mathrm{ArH}^{+}$for a very small unshielded cloud surrounded on all sides by the standard Draine UV ISRF is $9.9 \times 10^{-12} \mathrm{~s}^{-1}$. This value is very sensitive to the spectral distribution in the VUV 911-2400 A wavelength window, as shown in Table 2.

The corresponding rate relevant to the Crab nebula environment is $1.9 \times 10^{-9} \mathrm{~s}^{-1}$.

The dependence on the visual extinction $A_{\mathrm{V}}$ due to the attenuation of radiation by dust was derived from the Meudon PDR code both for incident isotropic and plane parallel radiations. We report the corresponding analytic functions for a semi-nfinite plane parallel cloud for different typical cases and for the Crab nebula environment. The constant numerical factor is close to half the value obtained for the unshielded environment. The dependence on $A_{V}$ is better represented with an $E_{2}$ function when isotropic radiation is impinging on the cloud. Nevertheless, we stressed that a direct integration of the product of photodissociation cross-sections by the actual radiation field allows photodestruction rates to be derived unambiguously. The actual value of the photodissociation rate is moderate, and destruction of $\mathrm{ArH}^{+}$ is mainly due to molecular $\mathrm{H}_{2}$. Then, both $\mathrm{ArH}^{+}$, and $\mathrm{OH}^{+}$, also detected in the Crab nebula (Barlow et al. 2013), are strongly dependent on the molecular fraction of the gas.

Acknowledgements. We thank M. J. Barlow and P. J. Owen for information on the Crab nebula environment. After the refereeing process of the paper, we became aware of an additional detection of $\mathrm{ArH}^{+}$in the diffuse ISM. We particularly thank D. Neufeld and J. H. Black for helpful exchanges and related informations. E.R. and J.L.B. acknowledge support from the CNRS National Programme PCMI (Physico-Chimie du Milieu Interstellaire). A.B.A. acknowledges support from the Deutsche Forschungsgemeinschaft.

\section{References}

Abgrall, H., Roueff, E., Launay, F., Roncin, J. Y., \& Subtil, J. L. 1993, J. Mol. Spectrosc., 157, 512

Abgrall, H., Roueff, E., \& Drira, I. 2000, A\&AS, 141, 297 


\section{E. Roueff et al.: Photodissociation of interstellar $\mathrm{ArH}^{+}$}

Alekseyev, A. B., Liebermann, H.-P., \& Buenker, R. J. 2003, in Recent advances in Relativistic Effects in Chemistry, eds. K. Hirao, \& Y. Ishikawa (Singapore World Scientific), 65

Alekseyev, A. B., Liebermann, H.-P., \& Buenker, R. J. 2007, Phys. Chem. Chem. Phys. (Incorporating Faraday Transactions), 9, 5088

Anicich, V. G. 2003, An Index of the Literature for Bimolecular Gas Phase Cation-Molecule Reaction Kinetics, Tech. rep., JPL Publication 03-19

Barlow, M. J., Swinyard, B. M., Owen, P. J., et al. 2013, Science, 342, 1343

Draine, B. T. 1978, ApJS, 36, 595

Goicoechea, J. R., \& Le Bourlot, J. 2007, A\&A, 467, 1

Habing, H. J. 1968, Bull. Astron. Inst. Netherlands, 19, 421

Hester, J. J. 2008, A\&A, 46, 127

Indriolo, N. 2013, in Advances in Cosmic Rays in Star-Forming Environments, eds. D. F. Torres, \& O. Reimer (Springer-Verlag), Astrophys. Space Sci. Proc., 34, 83

Jenkins, E. B. 2013, ApJ, 764, 25
Johns, J. W. C. 1984, J. Mol. Spectrosc., 106, 124

Le Petit, F., Nehmé, C., Le Bourlot, J., \& Roueff, E. 2006, ApJS, 164, 506 Loh, E. D., Baldwin, J. A., Ferland, G. J., et al. 2012, MNRAS, 421, 789

Mathis, J. S., Rumpl, W., \& Nordsieck, K. H. 1977, ApJ, 217, 425

McElroy, D., Walsh, C., Markwick, A. J., et al. 2013, A\&A, 550, A36

Mitchell, J. B. A., Novotny, O., LeGarrec, J. L., et al. 2005, J. Phys. B Atom. Mol. Phys., 38, L175

Neufeld, D. A., \& Wolfire, M. G. 2009, ApJ, 706, 1594

Rollins, R. P., \& Rawlings, J. M. C. 2012, MNRAS, 427, 2328

Saloman, E. B. 2010, J. Phys. Chem. Ref. Data, 39, 033101

Tielens, A. G. G. M., \& Hollenbach, D. 1985, ApJ, 291, 722

Šurkus, A. 2000, Spectrochim. Acta Part A: Molecular Spectroscopy, 56, 1979

van Dishoeck, E. F., Jonkheid, B., \& van Hemert, M. C. 2006, Faraday Discussions, 133, 231

Wakelam, V., Herbst, E., Loison, J.-C., et al. 2012, ApJS, 199, 21

Weingartner, J. C., \& Draine, B. T. 2001, ApJ, 548, 296 